\title{
Distributed Beamforming Design for Nonregenerative Two-Way Relay Networks with Simultaneous Wireless Information and Power Transfer
}

\author{
Keyun Liao, Sai Zhao (D), Yusi Long, Gaofei Huang, and Dong Tang \\ School of Mechanical and Electrical Engineering, Guangzhou University, Guangzhou, Guangdong Province, China \\ Correspondence should be addressed to Sai Zhao; zhaosai@gzhu.edu.cn
}

Received 12 April 2019; Revised 2 October 2019; Accepted 29 October 2019; Published 2 December 2019

Academic Editor: Daniele Pinchera

Copyright (c) 2019 Keyun Liao et al. This is an open access article distributed under the Creative Commons Attribution License, which permits unrestricted use, distribution, and reproduction in any medium, provided the original work is properly cited.

\begin{abstract}
This paper considers the distributed beamforming design for a simultaneous wireless information and power transfer (SWIPT) in twoway relay network, which consists of two sources, $K$ relay nodes and one energy harvesting $(\mathrm{EH})$ node. For such a network, assuming perfect channel state information (CSI) is available, and we study two different beamforming design schemes. As the first scheme, we design the beamformer through minimization of the average mean squared error (MSE) subject to the total transmit power constraint at the relays and the energy harvesting constraint at the $\mathrm{EH}$ receiver. Due to the intractable expression of the objective function, an upper bound of MSE is derived via the approximation of the signal-to-noise ratio (SNR). Based on the minimization of this upper bound, this problem can be turned into a convex feasibility semidefinite programming (SDP) and, therefore, can be efficiently solved using interior point method. To reduce the computational complexity, a suboptimal beamforming scheme is proposed in the second scheme, for which the optimization problem could be recast to the form of the Rayleigh-Ritz ratio and a closed-form solution is obtained. Numerical results are provided and analyzed to demonstrate the efficiency of our proposed beamforming schemes.
\end{abstract}

\section{Introduction}

Harvesting energy from environment has become a preferred strategy to overcome the key challenge of limited lifetime of devices equipped with battery in wireless networks, e.g., wireless sensor network (WSN) or Bluetooth low-energy mesh (BLE Mesh) network. As an efficient energy harvesting technique, simultaneous wireless information and power transfer (SWIPT) has gained considerable research attention in the academic field [1-6]. The concept of SWIPT was first introduced and a capacityenergy function was first proposed to characterize the fundamental tradeoff between simultaneous energy and information transfer in [1]. Considering SWIPT in multipleinput single-output (MISO) multicasting systems [2], the authors minimize the base station (BS) transmit power by jointly optimizing the beamforming vector at $\mathrm{BS}$ and the power splitting parameters of mobile station. In [3], considering a multiple-input multiple-output (MIMO) wireless broadcast scenario, where one receiver harvested energy and another receiver decoded information from the signals sent by a transmitter, two practical schemes, namely, time switching (TS) and power splitting (PS), were proposed. Motivated by the requirement of fifth generation, Xu et al. [4] investigated the cooperative SWIPT NOMA protocol design in single-input single-output (SISO) and multipleinput single-output (MISO). Meanwhile, the security of information transmission also became more and more important. Zhao et al. [5] considered the security of wireless network for SWIPT, which maximize the achievable secrecy rate subject to sum transmit power constraint at relays and energy harvesting (EH) constraint at the $\mathrm{EH}$ receiver. Aforementioned papers were investigated under perfect channel state information (CSI). In [6], the MIMO SWIPT system under the condition of imperfect CSI was studied.

Considering its advantage in enhancing spectral efficiency, two-way relay (TWR) network is extensively studied [7-10]. In the TWR network, two source nodes exchanged 
information through a relay node, and the number of required time slots is reduced from four to two with the aid of analog network coding [7]. In [8], a cooperative relay scheme for distributed amplify-and-forward (AF) relays working under the TWR protocol was considered, and a closed-form solution was obtained. Considering the user fairness, a linear precoding design for relay in multiple-pair two-way MIMO relay systems was investigated [9]. Considering security, cooperative beamforming in cognitive two-way relay networks was studied in [10]. For SWIPT two-way relay channels, the authors of [11-15] considered the relay beamforming design for SWIPT in a nonregenerative TWR network with the objective to maximize the sum rate of TWR. Wen et al. [12] studied the joint source and relay design for MIMO twoway relay networks with SWIPT, which minimized the total mean square error guaranteeing sufficient energy harvested at the sources. The authors of $[14,15]$ developed a novel distributed energy beamforming scheme for realizing SWIPT in the TWR network, where the former considered two source node exchanged information via an $\mathrm{EH}$ relay node and the latter considered multiple-user exchanged messages with the help of an energy-constrained relay node. Considering imperfect CSI and energy efficiency, the robust optimization schemes of MIMO two-way relay networks were considered under the constraint of energy harvesting [16]. In the IoT networks or sensor networks, most of the nodes may have limited volume and limited computation capacity with limited energy supply, some of the relay nodes are providing data communication service, while other idle node(s) could harvest energy to prolong its/their life cycle. That is why we consider multiple relays in two-way transmission, while at the same time, an EH is placed to harvest energy. And to our best knowledge, there is little work on this system model by now.

The main contributions of our paper are summarized as follows:

(1) We study the distributed beamforming design in SWIPT-based two-way multiple-relay networks. Differing from $[14,15]$, we consider the two-way SWIPT system with multiple relays, while only single relay is considered in $[14,15]$. Our goal is to minimize the upper bound of average MSE while satisfying the harvested energy requirements of the $\mathrm{EH}$ receiver and the total power budgets of the multiple relays.

(2) For the case of SWIPT-based two-way multiple-relay networks, the initial problem is a nonconvex problem. Through appropriate mathematical transformation, we could recast the initial problem into the semidefinite programming (SDP) problem. Then, we can obtain a quasi-optimal solution by applying semidefinite relaxation (SDR).

(3) To avoid the high computational complexity resulting from solving the SDP problem, we find the suboptimal solution by converting the initial problem into the form of the Rayleigh-Ritz quotient, which is of low complexity.

The rest of this paper is organized as follows: in Section 2, the system model and the problem formulation are illustrated.
Section 3 presents our proposed methods to obtain the solutions to the problem of beamforming design. Section 4 constructs complexity analysis of two schemes and provides simulation results. Finally, Section 5 has a conclusion about the paper.

Notations: boldface lowercase and uppercase letters denote vectors and matrices, respectively. The transpose, conjugate, conjugate transpose, Frobenius norm, and trace of matrix $\mathbf{A}$ are denoted as $\mathbf{A}^{T}, \mathbf{A}^{*}, \mathbf{A}^{H},\|\mathbf{A}\|$, and $\operatorname{tr}(\mathbf{A})$, respectively. $\operatorname{vec}(\mathbf{A})$ denotes to stack the columns of a matrix A into a single vector $\mathbf{a} . \otimes$ denotes the Kronecker product. $\lambda_{\max }(\mathbf{A})$ and $\lambda_{\min }(\mathbf{A})$ denote the maximum and minimum eigenvalues of $\mathbf{A}$, respectively. $\nu\{\mathbf{A}\}$ denotes the eigenvector of $\mathbf{A}$ associating with the maximum eigenvalue. By $\mathbf{A} \geqslant 0$, we mean that $\mathbf{A}$ is positive semidefinite. $\mathbf{I}$ is the identity matrix. $\mathscr{C} \mathscr{N}(0,1)$ denotes the distribution of a circularly symmetric complex Gaussian vector with mean vector 0 and variance 1 .

\section{System Model}

We consider a collaborative network consisting of two source nodes, $K$ AF relays using the two-way protocol, and one $\mathrm{EH}$ receiver as shown in Figure 1.

Each node in the whole network is equipped with a single antenna. All nodes are subject to the half-duplex mode. The two source nodes complete the information exchange with the help of $K$ distributed relay nodes within two time slots. In the first time slot, both $S_{1}$ and $S_{2}$ transmit symbols to all relays in the same frequency band simultaneously. The symbol received by the $k$ th relay can be describe as

$$
r_{k}=\sqrt{P_{1}} h_{1 k, f} s_{1}+\sqrt{P_{2}} h_{2 k, f} s_{2}+z_{k},
$$

where $P_{i}, i \in\{1,2\}$, are the transmit power at $S_{1}$ and $S_{2}$, respectively; $h_{i k, f}$ are the channel fading coefficient from $S_{i}$ to the $k$ th relay, where $k \in \mathscr{K}=\{1,2,3, \ldots, K\} ; s_{i}$ are the transmitted symbols from $S_{i}$, and $\mathbb{E}\left[\left|s_{i}\right|^{2}\right]=1$; and $z_{k} \sim \mathscr{C} \mathcal{N}\left(0, \sigma_{k}^{2}\right)$ is the additive white Gaussian noise (AWGN) at the $k$ th relay in the first time slot. We assume perfect channel state information (CSI) of all channels is known to both source nodes. The relay will perform the channel estimation from both sources to multiple relays and feedback the CSI to both source nodes. The EH nodes perform channel estimation from sources to the $\mathrm{EH}$ node in the first slot and perform channel estimation from relay to $\mathrm{EH}$ nodes in the second slot, then feedback to the sources. The synchronisation is performed by synchronisation signals sent from sources to relays and $\mathrm{EH}$ nodes so that all relays and $\mathrm{EH}$ can be perfectly synchronized. by

The symbol to be retransmitted by the $k$ th relay is given

$$
t_{k}=w_{k}\left(\sqrt{P_{1}} h_{1 k, f} s_{1}+\sqrt{P_{2}} h_{2 k, f} s_{2}+z_{k}\right)
$$

where $w_{k}$ is the complex beamforming weight at the $k$ th relay.

In the second time slot, the processed symbol $t_{k}$ is transmitted from the $k$ th relay to both $S_{1}$ and $S_{2}$ simultaneously, and the symbol received by $S_{1}$ and $S_{2}$ can be, respectively, written as 


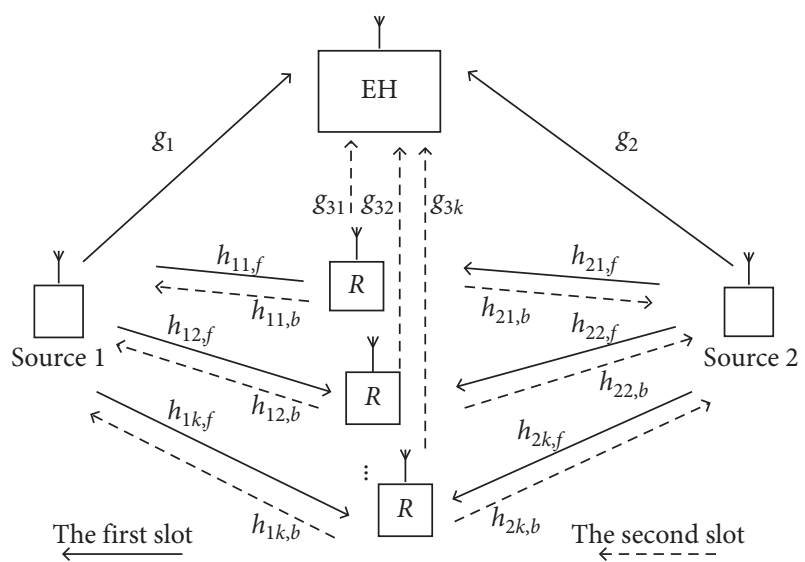

FIGURE 1: Illustration of the two-way relay network with SWIPT.

$$
\begin{aligned}
& y_{1}=\sum_{k=1}^{K} h_{1 k, b} t_{k}+n_{21}, \\
& y_{2}=\sum_{k=1}^{K} h_{2 k, b} t_{k}+n_{22},
\end{aligned}
$$

where $n_{2 i} \in \mathscr{C} \mathscr{N}\left(0, \sigma_{i}^{2}\right)$ is AWGN at $S_{i}$, respectively, in the second time slot and $h_{i k, b}$ are the channel fading coefficient from the $k$ th relay to $S_{i}, k \in \mathscr{K}=\{1,2,3, \ldots, K\}$.

Assume the channels are reciprocal during two time slots (e.g., under TDD mode), i.e., $h_{i k, f}=h_{i k, b}=h_{i k}$ for $i \in\{1,2\}$. Since each source node knows both the channels associated itself with the relay nodes and the weighted coefficients $w_{k}$, it can subtract the self-interference from itself which is similar to [11]. After this operation, (3) and (4) become

$$
\begin{aligned}
& \tilde{y}_{1}=\sqrt{P_{2}} \sum_{k=1}^{K} h_{1 k} w_{k} h_{2 k} s_{2}+\sum_{k=1}^{K} h_{1 k} w_{k} z_{k}+n_{21}, \\
& \tilde{y}_{2}=\sqrt{P_{1}} \sum_{k=1}^{K} h_{2 k} w_{k} h_{1 k} s_{1}+\sum_{k=1}^{K} h_{2 k} w_{k} z_{k}+n_{22} .
\end{aligned}
$$

The signal received by the $\mathrm{EH}$ receiver within two time slots can be, respectively, written as

$$
\begin{aligned}
e_{1}= & \sqrt{P_{1}} g_{1} s_{1}+\sqrt{P_{2}} g_{2} s_{2}+n_{\mathrm{EH}}, \\
e_{2}= & \sqrt{P_{1}} \sum_{k=1}^{K} g_{3 k} w_{k} h_{1 k} s_{1}+\sqrt{P_{2}} \sum_{k=1}^{K} g_{3 k} w_{k} h_{2 k} s_{2} \\
& +\sum_{k=1}^{K} g_{3 k} w_{k} z_{k}+n_{\mathrm{EH}},
\end{aligned}
$$

where $g_{i}$ denotes the channel fading coefficient from source node $S_{1}$ to the $\mathrm{EH}$ receiver, and $g_{3 k}, k \in \mathscr{K}$, denotes the channel fading coefficient from the $k$ th relay to the $\mathrm{EH}$ receiver, and $n_{\mathrm{EH}} \sim C N\left(0, \sigma_{\mathrm{EH}}^{2}\right)$ denotes AWGN at $\mathrm{EH}$ receiver. For simplicity, the noise powers at two sources, $K$ relays, and $\mathrm{EH}$ receiver, if not specified, are $\sigma_{1}^{2}=\sigma_{2}^{2}=\sigma_{k}^{2}=\sigma_{\mathrm{EH}}^{2}=\sigma^{2}$.

The harvested energy at the $\mathrm{EH}$ receiver during two time slots is given, respectively:

$$
\begin{aligned}
& E_{1}=P_{1}\left|g_{1}\right|^{2}+P_{2}\left|g_{2}\right|^{2}, \\
& E_{2}=P_{1}\left|\sum_{k=1}^{K} g_{3 k} w_{k} h_{1 k}\right|^{2}+P_{2}\left|\sum_{k=1}^{K} g_{3 k} w_{k} h_{2 k}\right|^{2}+\sigma^{2} \sum_{k=1}^{K}\left|g_{3 k} w_{k}\right|^{2} .
\end{aligned}
$$

In this paper, we will design a distributed relay beamforming to minimize the average MSE of bidirectional transmissions of two source nodes subject to the $\mathrm{EH}$ constraint and the total relay power constraint.

The MSE of each received symbol in traditional multipleinput multiple-output (MIMO) systems can be expressed by its signal-to-noise ratio $(\mathrm{SNR})$ as $\mathrm{MSE}=1 /(\mathrm{SNR}+1)$ if the Wiener filter is used at the receiver [17]. In two-way relay networks, the average MSE of the received symbols for bidirectional transmissions of two source nodes in (5) and (6) can be described as

$$
\mathrm{MSE}=0.5\left(1+\mathrm{SNR}_{1}\right)^{-1}+0.5\left(1+\mathrm{SNR}_{2}\right)^{-1}
$$

where $\mathrm{SNR}_{i}$ are the SNR for the received symbols for $S_{1}$ and $S_{2}$, respectively.

To simplify equation (9), the average MSE can be tightly approximated in the high SNR regime as

$$
\widetilde{\mathrm{MSE}}=0.5\left(\mathrm{SNR}_{1}\right)^{-1}+0.5\left(\mathrm{SNR}_{2}\right)^{-1} \geq \mathrm{MSE},
$$

where

$$
\begin{aligned}
& \mathrm{SNR}_{1}=\frac{P_{2}\left|\sum_{k=1}^{K} h_{1 k} w_{k} h_{2 k}\right|^{2}}{\sigma^{2}\left(1+\sum_{k=1}^{K}\left|h_{1 k}\right|^{2}\left|w_{k}\right|^{2}\right)}, \\
& \mathrm{SNR}_{2}=\frac{P_{1}\left|\sum_{k=1}^{K} h_{2 k} w_{k} h_{1 k}\right|^{2}}{\sigma^{2}\left(1+\sum_{k=1}^{K}\left|h_{2 k}\right|^{2}\left|w_{k}\right|^{2}\right)} .
\end{aligned}
$$

It is noted that $\widetilde{\mathrm{MSE}}$ is the upper bound of MSE. Based on $\widetilde{\mathrm{MSE}}$, the upper bound of MSE minimization problem can be formulated as

$$
\begin{array}{ll}
\min _{w_{k}} & \widetilde{\mathrm{MSE}}, \\
\text { s.t } \quad & \sum_{k=1}^{K}\left|w_{k}\right|^{2}\left(P_{1}\left|h_{1 k}\right|^{2}+P_{2}\left|h_{2 k}\right|^{2}+\sigma^{2}\right) \leq P_{3}, \\
& E_{1}+E_{2} \geq Q .
\end{array}
$$

It is noted that the optimization problem of the upper bound of MSE is a nonconvex problem due to the nonconvex of objective function and $\mathrm{EH}$ constraint. In the following section, we propose schemes to solve problem (12a)-(12c).

\section{Beamforming Design for Multiple Relays}

Firstly, the objective function in problem (12a)-(12c) can be rewritten as

$$
\widetilde{\mathrm{MSE}}=\frac{0.5\left(\sigma^{2}+\sigma^{2} \mathbf{w}^{H} \mathbf{B}_{1}^{H} \mathbf{B}_{1} \mathbf{w}\right)}{P_{1} \mathbf{w}^{H} \mathbf{g}_{e}^{*} \mathbf{g}_{e}^{T} \mathbf{w}}+\frac{0.5\left(\sigma^{2}+\sigma^{2} \mathbf{w}^{H} \mathbf{B}_{2}^{H} \mathbf{B}_{2} \mathbf{w}\right)}{P_{2} \mathbf{w}^{H}\left(\mathbf{g}_{e}^{*} \mathbf{g}_{e}^{T}\right) \mathbf{w}},
$$


where

$$
\begin{aligned}
& \mathbf{w} \triangleq\left(w_{1}, \ldots, w_{k}, \ldots, w_{K}\right)^{T}, \\
& \mathbf{B}_{1} \triangleq \operatorname{diag}\left\{\left(h_{21}, \ldots, h_{2 k}, \ldots, h_{2 K}\right)^{T}\right\}, \\
& \mathbf{B}_{2} \triangleq \operatorname{diag}\left\{\left(h_{11}, \ldots, h_{1 k}, \ldots, h_{1 K}\right)^{T}\right\}, \\
& \mathbf{g}_{e} \triangleq\left(h_{11} h_{21}, \ldots, h_{1 k} h_{2 k}, \ldots, h_{1 K} h_{2 K}\right)^{T} .
\end{aligned}
$$

Secondly, it is noted that the optimal beamforming vector $\mathbf{w}$ for problem (12a)-(12c) should guarantee that the total transmit power constraint (12b) at relays is active, i.e., (12b) can be written equivalently as

$$
\mathbf{w}^{H} \frac{\mathbf{B}_{3}}{\mathbf{P}_{3}} \mathbf{w}=1,
$$

where $\mathbf{B}_{3} \triangleq \operatorname{diag}\left\{\left(P_{1}\left|h_{11}\right|^{2}+P_{2}\left|h_{21}\right|^{2}+\sigma^{2}, \ldots, P_{1}\left|h_{1 k}\right|^{2}+P_{2}\right.\right.$ $\left.\left.\left|h_{2 k}\right|^{2}+\sigma^{2}, \ldots, P_{1}\left|h_{1 K}\right|^{2}+P_{2}\left|h_{2 K}\right|^{2}+\sigma^{2}\right)^{T}\right\}$.

Thirdly, substituting (15) into (12c), the constraint in (12c) can be recast as

$$
\mathbf{w}^{H}\left(P_{1} \mathbf{g}_{h 1}^{*} \mathbf{g}_{h 1}^{T}+P_{2} \mathbf{g}_{h 2}^{*} \mathbf{g}_{h 2}^{T}+\sigma^{2} \mathbf{g}_{3} \mathbf{g}_{3}^{H}-\frac{\widetilde{Q}}{P_{3}} \mathbf{B}_{3}\right) \mathbf{w} \geq 0,
$$

where

$$
\begin{aligned}
\mathbf{g}_{h 1} & \triangleq\left(g_{31} h_{11}, \ldots, g_{3 K} h_{1 K}\right)^{T}, \\
\mathbf{g}_{h 2} & \triangleq\left(g_{31} h_{21}, \ldots, g_{3 K} h_{2 K}\right)^{T}, \\
\widetilde{Q} & \triangleq Q-E_{1}, \\
\mathbf{g}_{3} & \triangleq \operatorname{diag}\left\{\left(g_{31}, \ldots g_{3 k}, \ldots g_{3 K}\right)^{T}\right\},
\end{aligned}
$$

and $Q$ denotes the threshold of the harvested energy at the $\mathrm{EH}$ receiver.

We choose $0 \leq Q \leq Q_{\max }$, where

$$
Q_{\max }=E_{1}+\lambda_{\max }\left(\mathbf{B}_{3}^{-1} \mathbf{B}_{4}\right) P_{3},
$$

where $\mathbf{B}_{4}=P_{1} \mathbf{g}_{h 1}^{*} \mathbf{g}_{h 1}^{T}+P_{2} \mathbf{g}_{h 2}^{*} \mathbf{g}_{h 2}^{T}+\sigma^{2} \mathbf{g}_{3} \mathbf{g}_{3}^{H}$. And $\lambda_{\max }(\cdot)$ indicates the maximum eigenvalue of a matrix. Substituting (15) into (13), ignoring the coefficient 0.5 , problem (12a)(12c) can be reformulated as

$$
\begin{array}{ll}
\min _{\mathbf{w}} & \frac{\mathbf{w}^{H}\left(\left(\sigma^{2} \mathbf{B}_{3} / P_{1} P_{3}\right)+\left(\sigma^{2} \mathbf{B}_{1}^{H} \mathbf{B}_{1} / P_{1}\right)+\left(\sigma^{2} \mathbf{B}_{3} / P_{2} P_{3}\right)+\left(\sigma^{2} \mathbf{B}_{2}^{H} \mathbf{B}_{2} / P_{2}\right)\right) \mathbf{w}}{\mathbf{w}^{H}\left(\mathbf{g}_{e}^{*} \mathbf{g}_{e}^{T}\right) \mathbf{w}}, \\
\text { s.t } \quad & \mathbf{w}^{H}\left(\frac{\mathbf{B}_{3}}{P_{3}}\right) \mathbf{w}=1, \\
& \mathbf{w}^{H}\left(\mathbf{B}_{4}-\frac{\widetilde{Q}}{P_{3}} \mathbf{B}_{3}\right) \mathbf{w} \geq 0 .
\end{array}
$$

In optimization problem (19a)-(19c), we have transformed the inequality constraint of total relay transmit power into an equality constraint. This is because the objective function and the constraint in (15) is homogeneous in $\mathbf{w}$. An arbitrary positive scaling of $\mathbf{w}$ has no effect on the value of the objective function. Furthermore, if $\mathbf{w}$ satisfies the constraint, an arbitrary positive scaling of $\mathbf{w}$ also satisfies the constraint.

3.1. The Local Optimal Beamforming Scheme. The optimization problem (19a)-(19c) is a fractional quadratically constrained quadratic problem (QCQP) which is intractable. We transform the problem (19a)-(19c) into the following fractional semidefinite programming (SDP) problem [18]:

$$
\begin{array}{ll}
\min _{\mathbf{W}} & \frac{\operatorname{Tr}\left(\mathbf{C}_{1} \mathbf{W}\right)}{\operatorname{Tr}\left(\mathbf{C}_{2} \mathbf{W}\right)}, \\
\text { s.t } \quad & \operatorname{Tr}\left(\mathbf{C}_{3} \mathbf{W}\right)=1, \\
& \operatorname{Tr}\left(\mathbf{C}_{4} \mathbf{W}\right) \geq 0,
\end{array}
$$

where

$$
\begin{aligned}
& \mathbf{C}_{1}=\sigma^{2}\left(\frac{\mathbf{B}_{3}}{P_{1} P_{3}}+\frac{\mathbf{B}_{1}^{H} \mathbf{B}_{1}}{P_{1}}+\frac{\mathbf{B}_{3}}{P_{2} P_{3}}+\frac{\mathbf{B}_{2}^{H} \mathbf{B}_{2}}{P_{2}}\right), \\
& \mathbf{C}_{2}=\mathbf{g}_{e}^{*} \mathbf{g}_{e}^{T}, \\
& \mathbf{C}_{3}=\frac{\mathbf{B}_{3}}{P_{3}}, \\
& \mathbf{C}_{4}=\mathbf{B}_{4}-\frac{\widetilde{Q}}{P_{3}} \mathbf{B}_{3}, \\
& \mathbf{W}=\mathbf{w w}^{H} .
\end{aligned}
$$

It is noted that the rank one constraint $\operatorname{Rank}(\mathrm{W})=1$ has been removed within the problem (20a)-(20c), and the problem (20a)-(20c) is a quasi-convex optimization problem.

We have the following proposition.

Proposition 1. The fractional SDP problem (20a)-(20c) can be written equivalent as 


$$
\begin{array}{ll}
\min _{\mathbf{W}} & \operatorname{Tr}\left(\mathbf{C}_{1} \mathbf{W}\right), \\
\text { s.t } & \operatorname{Tr}\left(\mathbf{C}_{2} \mathbf{W}\right)=1, \\
& \operatorname{Tr}\left(\mathbf{C}_{3} \mathbf{W}\right)=1, \\
& \operatorname{Tr}\left(\mathbf{C}_{4} \mathbf{W}\right) \geq 0 .
\end{array}
$$

Proof of Proposition 1. Suppose that $\left(t_{1}, \mathbf{W}_{1}^{o}\right)$ and $\left(t_{2}, \mathbf{W}_{2}^{o}\right)$ are the optimal value and optimal solution to the problems (20a)-(20c) and (22a)-(22d), respectively. It is obvious that $\mathbf{W}_{2}^{o}$ is feasible to problem (20a)-(20c). When $\mathbf{W}=\mathbf{W}_{2}^{o}$, the objective value of (20a)-(20c) is $t_{2}$. Therefore, $t_{2} \geq t_{1}$.

On the contrary, it is easy to prove that $\mathbf{W}_{1}^{o} / \operatorname{Tr}\left(\mathbf{C}_{2} \mathbf{W}_{1}^{o}\right)$ is the feasible to problem (22a)-(22d). When $\mathbf{W}=\mathbf{W}_{1}^{o} / \mathrm{Tr}$ $\left(\mathbf{C}_{2} \mathbf{W}_{1}^{o}\right)$, the objective value of $(22 \mathrm{a})-(22 \mathrm{~d})$ is $t_{1}$. Therefore, $t_{1} \geq t_{2}$. That means, problems (20a)-(20c) and (22a)-(22d) have the same optimal values, e.g., $t_{1}=t_{2}$. Therefore, problem (20a)-(20c) is equivalent to (22a)-(22d).

Suppose that $\mathbf{W}^{o}$ is the optimal solution to the problem (22a)-(22d). If $\operatorname{Rank}\left(\mathbf{W}^{o}\right)=1$, the optimal solution to problem (20a)-(20c) is $\mathbf{W}_{1}^{o}$ which can be obtained by the eigenvalue decomposition technique, i.e., $\mathbf{W}_{1}^{o}=\sqrt{c} \mathbf{u}$, where $c$ and $\mathbf{u}$ denote the eigenvalue and eigenvector of $\mathbf{W}^{o}$, respectively. In the case of $\operatorname{Rank}\left(\mathbf{W}^{o}\right) \geq 2$, we apply the Gaussian randomization technique [9] to obtain the final solution $\mathbf{W}_{1}^{o}$ of problem (19a)-(19c) from the solution of problem (20a)-(20c). To obtain the final solution of initial problem (12a)-(12c), we also need to scale $\mathbf{W}_{1}^{o}$ to satisfy the relay power constraint.

3.2. The Closed-Form Beamforming Scheme. To reduce the computational complexity, we propose a suboptimal closedform solution to the problem (19a)-(19c). Define $\mathbf{U}$ as a matrix which consists of the $a(1 \leq a \leq K)$ eigenvectors of matrix $\mathbf{C}_{4}$ which are associated with the eigenvalues being greater than zero. We have

$$
\mathbf{w}=\mathbf{U} \mathbf{x},
$$

where $\mathbf{x}$ is an arbitrary vector such that

$$
\mathbf{w}^{H} \mathbf{C}_{4} \mathbf{w} \geq 0 .
$$

Substituting (24) into problem (19a)-(19c), we have the equivalent maximization problem:

$$
\begin{array}{ll}
\max _{\mathbf{x}} & \frac{\mathbf{x}^{H} \mathbf{D}_{2} \mathbf{x}}{\mathbf{x}^{H} \mathbf{D}_{1} \mathbf{x}} \\
\text { s.t } & \mathbf{x}^{H} \mathbf{D}_{3} \mathbf{x}=1,
\end{array}
$$

where

$$
\begin{aligned}
& \mathbf{D}_{1}=\mathbf{U}^{H} \mathbf{C}_{1} \mathbf{U}, \\
& \mathbf{D}_{2}=\mathbf{U}^{H} \mathbf{C}_{2} \mathbf{U}, \\
& \mathbf{D}_{3}=\mathbf{U}^{H} \mathbf{C}_{3} \mathbf{U} .
\end{aligned}
$$

Introduce $\widetilde{\mathbf{x}}=\mathbf{D}_{3}^{1 / 2} \mathbf{x}$ so that $\mathbf{x}=\mathbf{D}_{3}^{-(1 / 2)} \widetilde{\mathbf{x}}$. Substituting $\mathbf{x}$ into (25), we are able to transform problem (25) into the following form:

$$
\begin{array}{ll}
\max _{\widetilde{\mathbf{x}}} & \frac{\widetilde{\mathbf{x}}^{H} \mathbf{G}_{2} \widetilde{\mathbf{x}}}{\widetilde{\mathbf{x}}^{H} \mathbf{G}_{1} \widetilde{\mathbf{x}}}, \\
\text { s.t } & \widetilde{\mathbf{x}}^{H} \widetilde{\mathbf{x}}=1,
\end{array}
$$

where

$$
\begin{aligned}
& \mathbf{G}_{1}=\left(\mathbf{D}_{3}^{-(1 / 2)}\right)^{H} \mathbf{D}_{1} \mathbf{D}_{3}^{-(1 / 2)}, \\
& \mathbf{G}_{2}=\left(\mathbf{D}_{3}^{-(1 / 2)}\right)^{H} \mathbf{D}_{2} \mathbf{D}_{3}^{-(1 / 2)} .
\end{aligned}
$$

It is noted that the problem (27a) and (27b) is a generalized Rayleigh quotient problem [16]. Defining $\mathbf{q}=\mathbf{G}_{1}^{1 / 2} \widetilde{\mathbf{x}}$, we can rewrite (27a) and (27b) as

$$
\max _{\mathbf{q}} \frac{\mathbf{q}^{H}\left(\mathbf{G}_{1}^{H}\right)^{1 / 2} \mathbf{G}_{2} \mathbf{G}_{1}^{1 / 2} \mathbf{q}}{\mathbf{q}^{H} \mathbf{q}} .
$$

Thus, the optimal solution to (29) can be obtained as

$$
\begin{aligned}
\mathbf{q} & =v_{\max }\left\{\left(\mathbf{G}_{1}^{H}\right)^{1 / 2} \mathbf{G}_{2} \mathbf{G}_{1}^{1 / 2}\right\} \\
& =\left(\mathbf{G}_{1}^{H}\right)^{1 / 2}\left(\mathbf{D}_{3}^{H}\right)^{-(1 / 2)} \mathbf{U}^{H} \mathbf{g}_{e}^{*},
\end{aligned}
$$

where $v_{\max }\{\mathbf{A}\}$ denotes the eigenvector corresponding to the largest eigenvalue of matrix A. Using (30), we have

$$
\begin{aligned}
\tilde{x} & =\mathbf{G}_{1}^{1 / 2} \mathbf{q} \\
& =\kappa \mathbf{G}_{1}^{1 / 2}\left(\mathbf{G}_{1}^{H}\right)^{1 / 2}\left(\mathbf{D}_{3}^{H}\right)^{-(1 / 2)} \mathbf{U}^{H} \mathbf{g}_{e}^{*},
\end{aligned}
$$

where $\kappa=1 /\left\|\mathbf{G}_{1}^{1 / 2}\left(\mathbf{G}_{1}^{H}\right)^{1 / 2}\left(\mathbf{D}_{3}^{H}\right)^{-(1 / 2)} \mathbf{U}^{H} \mathbf{g}_{e}^{*}\right\|$ is used to normalize $\tilde{\mathbf{x}}$ that satisfies the constraint (27b). Substituting (31) into $\mathbf{x}=\mathbf{D}_{3}^{-(1 / 2)} \tilde{\mathbf{x}}$ and combining (23), we have

$$
\begin{aligned}
\mathbf{w} & =\kappa \mathbf{U D}_{3}^{-(1 / 2)} \mathbf{G}_{1}^{1 / 2}\left(\mathbf{G}_{1}^{H}\right)^{1 / 2}\left(\mathbf{D}_{3}^{H}\right)^{-(1 / 2)} \mathbf{U}^{H} \mathbf{g}_{e}^{*} \\
& =\kappa\left(\mathbf{U D}_{3}^{-(1 / 2)} \mathbf{G}_{1}^{1 / 2}\right)\left(\mathbf{U D}_{3}^{-(1 / 2)} \mathbf{G}_{1}^{1 / 2}\right)^{H} \mathbf{g}_{e}^{*} .
\end{aligned}
$$

\section{Simulation Results}

In the section, we present the simulation results of our proposed beamforming schemes. We assume that the channel from the source node $S_{i}$ to the $k$ th relay is modeled as $h_{i k}=d_{i, k}^{-(\chi / 2)} \widetilde{h}_{i k}$ and the channel from the source node $S_{i}$ to the EH receiver is modeled as $g_{i}=d_{i, \mathrm{EH}}^{-(\gamma / 2)} \tilde{g}_{i}$. The channel from the $k$ th relay to the $\mathrm{EH}$ receiver is modeled as $g_{3 k}=d_{k, \mathrm{EH}}^{-(\chi / 2)} \widetilde{g}_{3 k}$, where $d_{i, k}$ is the distance between $S_{i}$ and the $k \mathrm{th}$ relay; $d_{i, \mathrm{EH}}$ is the distance between $S_{i}$ and the EH receiver; $d_{k, \mathrm{EH}}$ is the distance between the $k$ th relay and the $\mathrm{EH}$ receiver; $\chi=3$ is the path loss exponent; and $\widetilde{h}_{i k}, \widetilde{g}_{i}$, and $\widetilde{g}_{3 k}$ are the independent identically distributed (i.i.d.) Rayleigh fading channels, where $i \in\{1,2\}, k \in \mathscr{K}$. We consider a symmetric network with $d_{1, \mathrm{EH}}=d_{2, \mathrm{EH}}=8 \mathrm{~m}$, and $K$ relays are symmetrically placed at the two sides of the EH receiver with $d_{2 j+1, E H}=d_{2 j, E H}=j * 6 \mathrm{~m}$, where $j \in\{1, \ldots, K / 2\}$. Through all the simulations, the transmit power of two 
sources, if not specified, are $P_{1}=P_{2}=10 \mathrm{~dB}$, and the noise variance is set to be $\sigma^{2}=-25 \mathrm{~dB}$. The number of Monte Carlo simulations is set to 1000 .

In the simulation, different beamforming schemes are considered including the proposed two schemes, i.e., local optimal scheme (denoted as "Local-optimal"), closed-form scheme (denoted as "Closed-form"), and the global optimal scheme(denoted as "Global-optimal") in [11]. The "Globaloptimal" scheme obtains the global optimal solution based on the two-dimensional search and semidefinite programming.

Figure 2 shows the average sum rate of both $S_{1}$-to- $S_{2}$ and $S_{2}$-to- $S_{1}$-directional transmissions versus $P_{3}$ in the underlying networks, comparing the "Global-optimal" scheme, "Local-optimal" scheme, and "Closed-form" scheme. Here, we consider the threshold of the EH constraint is $Q=0.5 Q_{\max }$ and the number of relay is $K=4$. We observe that the "Global-optimal" scheme outperforms the proposed schemes "Local-optimal" scheme and "Closedform" scheme. Meanwhile, the "Local-optimal" scheme has better performance than the "Closed-form" scheme. It is also noted that as $P_{3}$ increases, the average sum rates increase for three schemes.

In Figure 3, we compare three schemes in terms of average sum rate versus various $\mathrm{EH}$ thresholds, where $Q=\tau Q_{\max }, K=4$, and $P_{3}=25 \mathrm{~dB}$. It is noted that, for different EH thresholds, the "Global-optimal" scheme has the best performance, and the "Local-optimal" scheme outperforms the "Closed-form" scheme. It is also noted that as $\tau$ increases, the average sum rates decrease for all three schemes.

In Figure 4, we present the average sum rate comparison of three schemes for different number of relays when the transmit power of relay $P_{3}=40 \mathrm{~dB}$ and the threshold of $\mathrm{EH}$ constraint $Q=0.1 Q_{\max }$. It is found that the "Global-optimal" scheme has the best performance and the "Closedform" scheme has the worst performance. With the increase of the number of relays, the average sum rate of the three schemes increases.

Here, we provide the complexity analysis of the "Globaloptimal" scheme, "Local-optimal" scheme, and "Closedform" scheme. According to [20], the complexity of obtaining an optimal solution for an SDP problem can be approximated as $\mathcal{O}\left(\max (m, n)^{4} \sqrt{n} \log (1 / \varepsilon)\right)$, where $\varepsilon$ denotes the solution accuracy and $m$ and $n$ are the number of constraints and the dimension of the matrix, respectively.

(i) For the "Global-optimal" scheme, $m=4$ and $n=K$. Thus, the computational complexity of obtaining an global optimal solution is $\mathcal{O}\left(\left(T \max (m, K)^{4}\right.\right.$ $\left.\sqrt{n} \log (1 / \mathcal{E}))+n_{r d}\right)$ where $T$ is the average iteration number of the two-dimensional search and $n_{r d}$ is the complexity of the Gaussian randomization.

(ii) For the "Local-optimal" scheme, the computational complexity is from the SDP problem (22a)-(22d), $m=3$ and $n=K$. Therefore, the overall computational complexity of (22a)-(22d) is $\mathcal{O}(\max$ $\left.(m, K)^{4} \sqrt{n} \log (1 / \mathcal{\varepsilon})\right)+n_{r d}$.

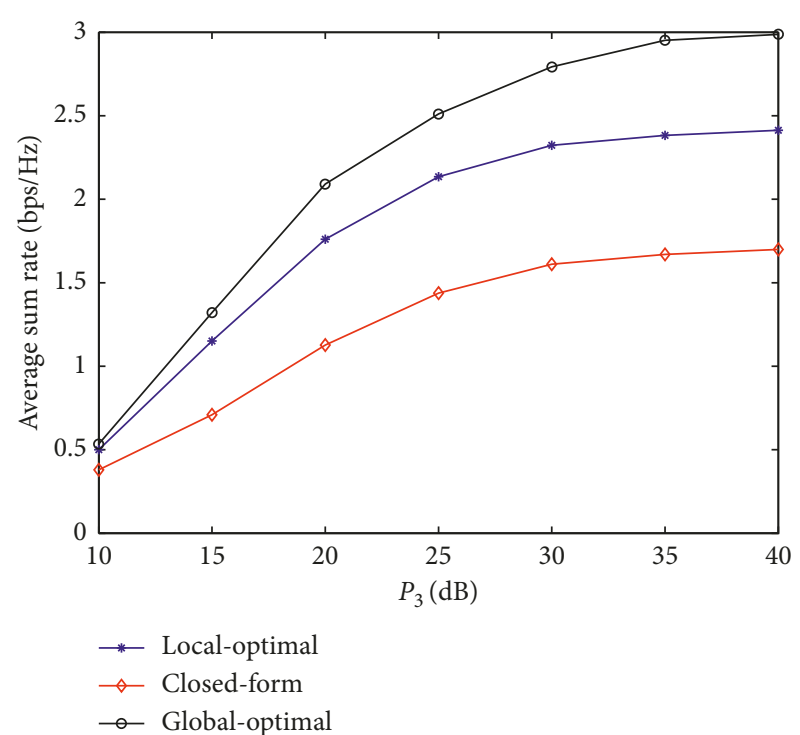

Figure 2: Average sum rate versus $P_{3}$ when the $\mathrm{EH}$ threshold $\mathrm{Q}=$ $0.5 Q_{\max }$ and $K=4$.

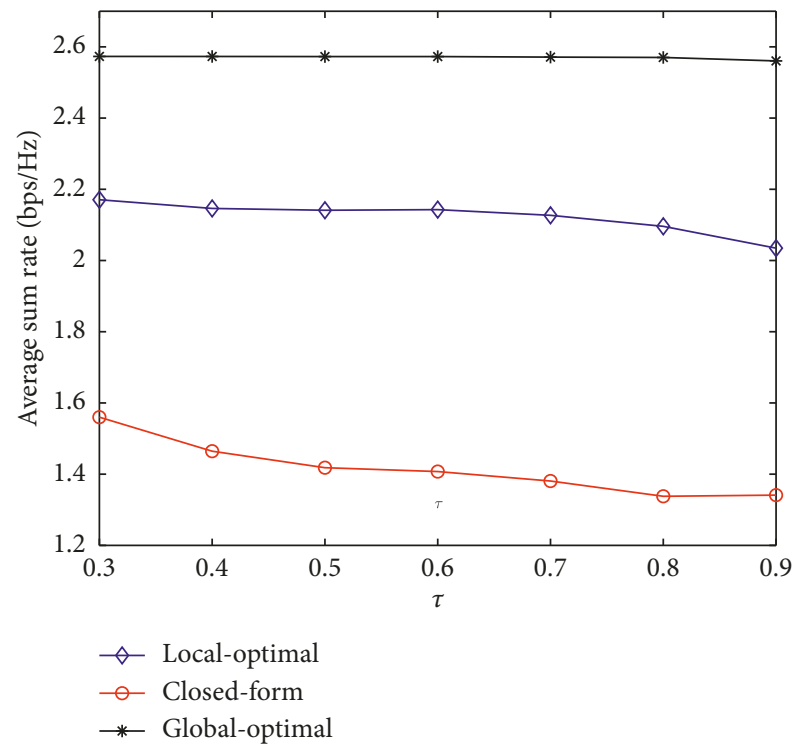

FIgURE 3: Average sum rate versus $\tau$ when the transmit power to noise power ratio of relay $P_{3}=25 \mathrm{~dB}$ and $K=4$.

(iii) For the "Closed-form" scheme, the main computational complexity is to solve the function of the problem (27a) and (27b). The computation of the generalized eigenvector of matrix pair $\left(G_{2}, G_{1}\right)$ requires $\mathcal{O}\left(a^{3}\right)$ arithmetic operations. Thus, the total computational complexity of the "Closed-form" scheme is $\mathcal{O}\left(a^{3}\right)$.

Obviously, the computational complexity of the "Globaloptimal" scheme is very high due to the two-dimensional search. The "Local-optimal" scheme has comparatively much lower computational complexity than the "Globaloptimal" scheme, and the "Closed-form" scheme has the lowest computational complexity. 


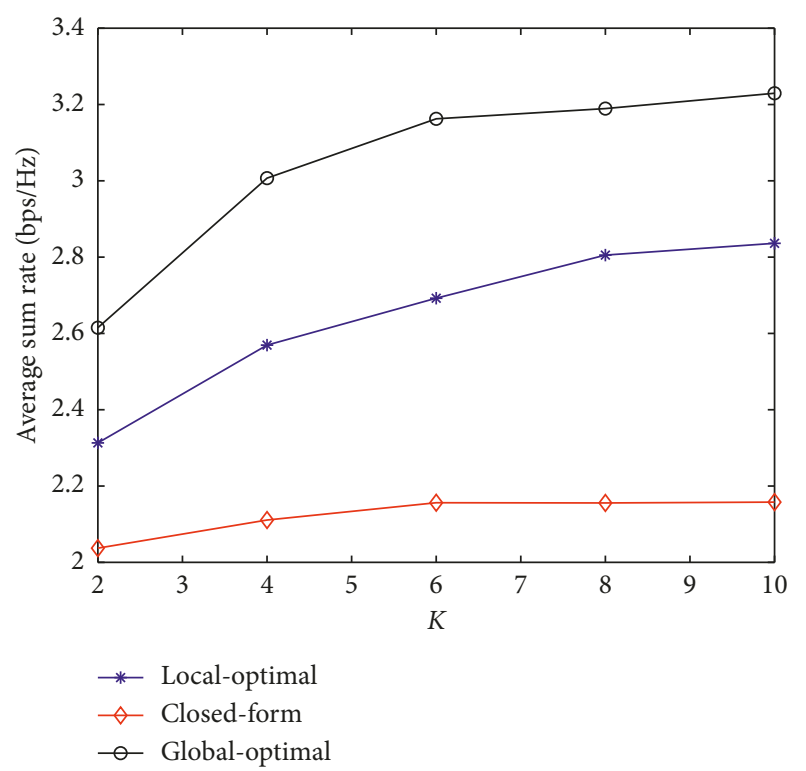

FIGURE 4: Average sum rate versus different number of relays when the EH threshold $Q=0.1 Q_{\max }$ and the transmit power at relays $P_{3}=40 \mathrm{~dB}$.

\section{Conclusion}

In this paper, we investigate the low complexity distributed beamforming scheme for the two-way multiple relays with SWIPT system. Though the "Global-optimal" scheme in [11] outperforms the proposed schemes, its computational complexity is very high compared to the two proposed schemes. The "Local-optimal" beamforming scheme has better performance than the "Closed-form" beamforming scheme; however, the computational complexity of the former is higher than that of the latter. Simulation results have shown the effectiveness of the proposed schemes. The multiple antennas equipped with each node in the system and more sophisticated EH model will be considered as an extension research of this paper.

\section{Data Availability}

No data were used to support this study.

\section{Conflicts of Interest}

The authors declare that they have no conflicts of interest.

\section{Acknowledgments}

This work was supported in part by the National Natural Science Foundation of China under grants 61902084, 61872098, and 61872102, Guangdong Natural Science Foundation under grant 2017A030310639, and Featured Innovation Project of Guangdong Education Department under grant 2018KTSCX174.

\section{References}

[1] L. R. Varshney, "Transporting information and energy simultaneously," in Proceedings of the 2008 IEEE International
Symposium on Information Theory, pp. 1612-1616, Toronto, Canada, July 2008.

[2] M. R. A. Khandaker and K.-K. Wong, "SWIPT in MISO multicasting systems," IEEE Wireless Communications Letters, vol. 3, no. 3, pp. 277-280, 2014.

[3] R. Zhang and C. K. Ho, "MIMO broadcasting for simultaneous wireless information and power transfer," IEEE Transactions on Wireless Communications, vol. 12, no. 5, pp. 1989-2001, 2013.

[4] Y. Xu, C. Shen, Z. Ding et al., "Joint beamforming and powersplitting control in downlink cooperative SWIPT NOMA systems," IEEE Transactions on Signal Processing, vol. 65, no. 18, pp. 4874-4886, 2017.

[5] S. Zhao, Q. Li, Q. Zhang, and J. Qin, "Optimal secure relay beamforming for non-regenerative multi-relay networks with energy harvesting constraint," Wireless Personal Communications, vol. 85, no. 4, pp. 2355-2365, 2015.

[6] C. Xing, N. Wang, J. Ni, Z. Fei, and J. Kuang, "MIMO beamforming designs with partial CSI under energy harvesting constraints," IEEE Signal Processing Letters, vol. 20, no. 4, pp. 363-366, 2013.

[7] R. Zhang, Y.-C. Liang, C. C. Chai, and S. Cui, "Optimal beamforming for two-way multi-antenna relay channel with analogue network coding," IEEE Journal on Selected Areas in Communications, vol. 27, no. 5, pp. 699-712, 2009.

[8] C. Li, L. Yang, and Y. Shi, "An asymptotically optimal cooperative relay scheme for two-way relaying protocol," IEEE Signal Processing Letters, vol. 17, no. 2, pp. 145-148, 2010.

[9] M. Tao and R. Wang, "Linear precoding for multi-pair twoway MIMO relay systems with max-min fairness," IEEE Transactions on Signal Processing, vol. 60, no. 10, pp. 53615370, 2012.

[10] Q. Li and L. Yang, "Beamforming for cooperative secure transmission in cognitive two-way relay networks," IEEE Transactions on Information Forensics and Security, vol. 15, no. 1, pp. 130-143, 2020.

[11] Q. Li, Q. Zhang, and J. Qin, "Beamforming in non-regenerative two-way multi-antenna relay networks for simultaneous wireless information and power transfer," IEEE Transactions on Wireless Communications, vol. 13, no. 10, pp. 5509-5520, 2014.

[12] Z. Wen, X. Liu, S. Zheng, and W. Guo, "Joint source and relay design for MIMO two-way relay networks with SWIPT," IEEE Transactions on Vehicular Technology, vol. 67, no. 1, pp. 822-826, 2018.

[13] X. Zhou and Q. Li, "Energy efficiency for SWIPT in MIMO two-way amplify-and-forward relay networks," IEEE Transactions on Vehicular Technology, vol. 67, no. 6, pp. 4910-4924, 2018.

[14] Z. Fang, X. Yuan, and X. Wang, "Distributed energy beamforming for simultaneous wireless information and power transfer in the two-way relay channel," IEEE Signal Processing Letters, vol. 22, no. 6, pp. 656-660, 2015.

[15] Z. Fang and X. Yuan, "Distributed energy beamforming and information transfer for multiway relay networks," IEEE Access, vol. 6, pp. 38977-38985, 2018.

[16] Q. Li and L. Yang, "Robust optimization for energy efficiency in mimo two-way relay networks with SWIPT," IEEE Systems Journal, pp. 1-12, 2019.

[17] D. P. Palomar, A unified framework for communications through MIMO channels, Ph.D. Tech, University of Catalonia, Signal Theory and Communications, Barcelona, Spain, 2003.

[18] S. Boyd and L. Vandenberghe, Convex Optimization, Cambridge University Press, Cambridge, UK, 2004. 
[19] X. Zhang, Matrix Analysis and Applications, Tsinghua University Press, Beijing, China, 2004.

[20] Z.-Q. Luo, W.-K. Ma, A. So, Y. Ye, and S. Zhang, "Semidefinite relaxation of quadratic optimization problems," IEEE Signal Processing Magazine, vol. 27, no. 3, pp. 20-34, 2010. 


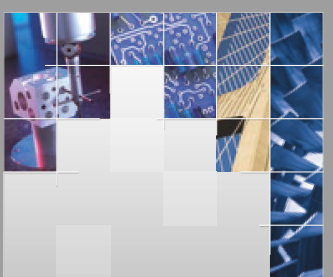

\section{Enfincering}
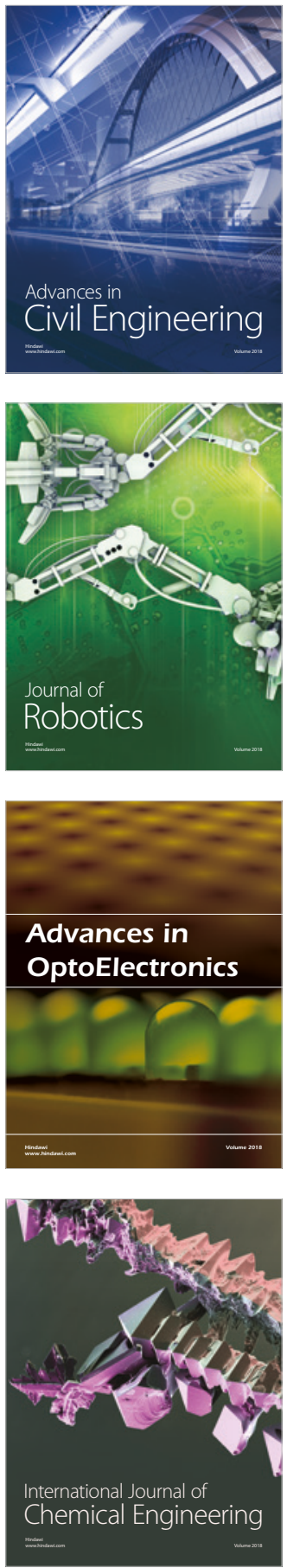

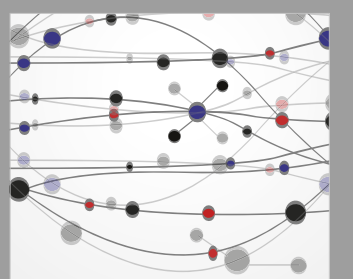

\section{Rotating \\ Machinery}

The Scientific World Journal

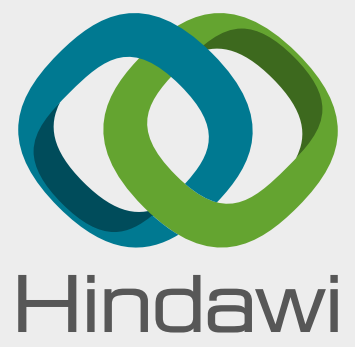

Submit your manuscripts at

www.hindawi.com
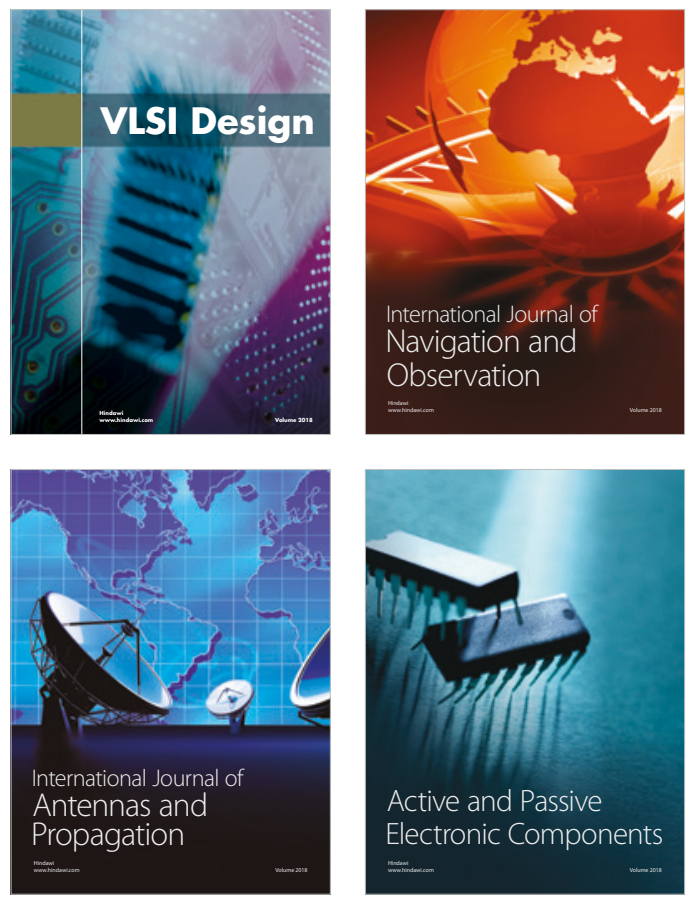
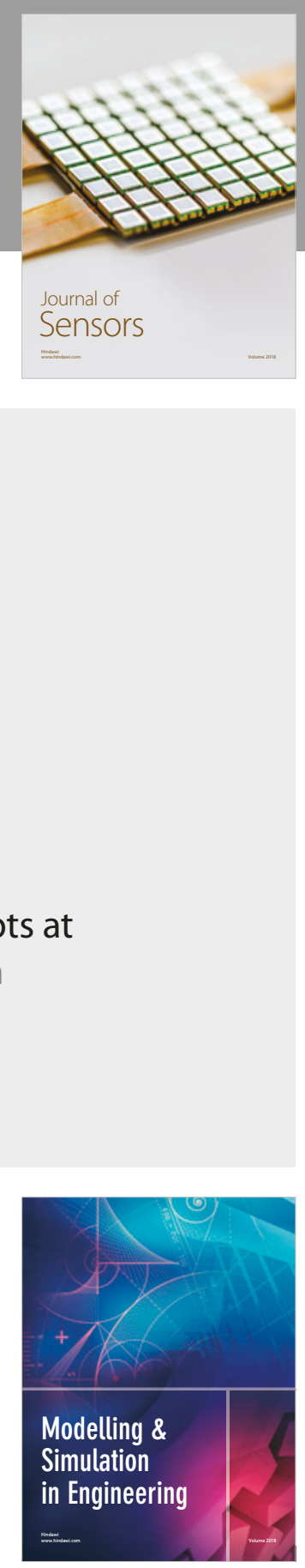

\section{Advances \\ Multimedia}
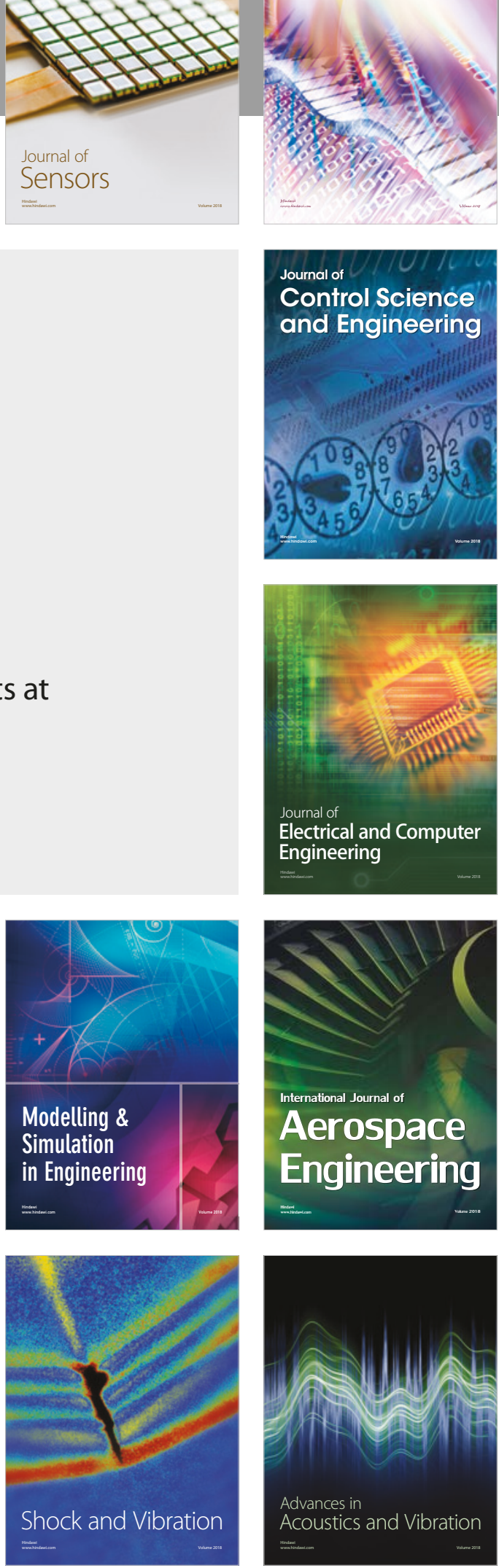